\title{
Epithelial Hes1 maintains gut homeostasis by preventing microbial dysbiosis
}

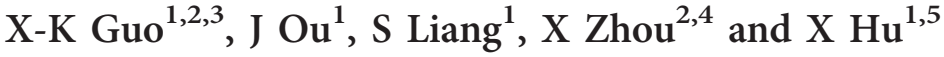

Recent advancements suggest that in addition to its roles in developmental processes, transcription repressor hairy and enhancer of split 1 (Hes1) also acts as a key regulator of inflammatory responses. A healthy gut microbiota ecology is critical for establishment of tissue homeostasis. However, the role of epithelial Hes1 in regulating intestinal microbiota ecology and intestinal homeostasis remains unexplored. Here we show that epithelial Hes1 deficiency leads to intestinal microbial dysbiosis and disturbed homeostasis. Both inducible Hes1 deletion and intestinal epithelial cell (IEC)-intrinsic Hes1 deletion resulted in loss of Bacteroidetes in ileum and increase of Escherichia coli and Akkermansia muciniphila in colon. Loss of Bacteroidetes closely correlated with decreased expression of commensal-dependent antimicrobial genes, leading to impaired resistance against pathogenic bacterial colonization. Moreover, Hes1 deficiency enhanced susceptibility to Dextran sodium sulphate-induced intestinal inflammation. Of note, transfer of Hes1-deficient-mouse-derived fecal microbiota promoted intestinal inflammation. The increase of $A$. muciniphila in colon was associated with Hes1-deficiency-induced unbalanced mucosal microhabitats. Thus, our results support that IEC-intrinsic Hes1 maintains gut homeostasis by preventing microbial dysbiosis partially through regulating mucosal microhabitats.

\section{INTRODUCTION}

The mammalian intestinal mucosal surfaces are under continuous exposure to trillions of commensal microorganisms (microbiota) whose compositions are critical for establishment of gut homeostasis. Specialized intestinal epithelial cells (IECs) constitute the gut epithelium forming a biochemical and physical barrier that maintains segregation of microbial communities from host tissues. ${ }^{1}$ Paneth cells, one of the epithelial secretory cell lineages, are important sources of antimicrobial proteins (AMPs) in the small intestine. ${ }^{2}$ Unlike Paneth cells, goblet cells mainly produce plentiful mucins that are essential for establishing a physical mucus barrier to prevent microbial contact with epithelia. ${ }^{3,4}$ Different lineages of IECs cope with the complex microbiota in a cooperative manner to maintain intestinal homeostasis. ${ }^{1}$ Aberrant microbiota composition, termed dysbiosis, can perturb normal immune regulatory networks, and is associated with human illnesses such as inflammatory bowel disease. ${ }^{5}$ A plethora of external and genetic factors, such as diet, antibiotics, AMPs, intestinal IgA, and mucus components, have been shown to play an important role in shaping gut microbiota. ${ }^{6-12}$

Although gut microbiota can be extensively shaped by external factors, it remains partially stable in adult intestine. For example, a longitudinal study shows that after severe perturbations by antibiotics, the colonic community is depleted to a lowdiversity composition but the membership and relative abundance are largely recovered to resemble the initial status after a prolonged period of time. ${ }^{13}$ It has been proposed that adult intestine may harbor reservoirs for bacterial cells that can be re-seeded into the intestinal lumen ${ }^{10}$ and thus alterations of endogenous genetic factors that influence mucosal reservoirs may have long-lasting effects on shaping microbiota ecology.

Hairy and enhancer of split 1 (Hes1) is a basic helix-loophelix transcriptional repressor that has key roles in the development of many organs and cell types. ${ }^{14}$ Genetic studies have revealed that Notch signaling controls the fate of IECs by inducing the expression of Hes genes and by repressing Atoh $1 .{ }^{15-18}$ However, in adult mice, Hes1 is not critically

${ }^{1}$ Institute for Immunology and School of Medicine, Tsinghua University, Beijing, China. ${ }^{2}$ Beijing Advanced Innovation Center for Food Nutrition and Human Health, China Agricultural University, Beijing, China. ${ }^{3}$ Tsinghua-Peking Center for Life Sciences, Tsinghua University, Beijing, China. ${ }^{4}$ College of Plant Protection, China Agricultural University, Beijing, China and ${ }^{5}$ Collaborative Innovation Center for Biotherapy, Tsinghua University, Beijing, China. Correspondence: X Hu. (xiaoyuhu@tsinghua.edu.cn) 
required for the development of intestinal IECs, mostly likely to be due to compensation by other Hes family members such as Hes 3 and Hes5. ${ }^{19}$ Thus, it is unclear what is the function of Hes 1 in adult intestinal epithelia. Recently, we have shown that myeloid expressed Hes1 is engaged in a negative feedback loop to modulate inflammatory responses, ${ }^{20-24}$ which suggests that Hes 1 may be involved in autoimmune and inflammatory conditions beyond developmental processes. Whether Hes1 regulates microbiota ecology and intestinal inflammation remains unexplored.

In this study, we assessed the role of Hes1 in regulating intestinal microbiota and homeostasis using two lines of conditional Hes1 knockout mice. Hes1-deficient mice displayed gut dysbiosis, attenuated epithelial barrier function, and increased susceptibility to pathogenic bacterial infection, as well as to Dextran sodium sulphate (DSS)-induced colonic inflammation. Moreover, susceptibility to intestinal inflammation was transferable by microbiota from Hes1-deficient hosts, which could be partially attributed to altered mucosal microhabitats. Taken together, we show that epithelial Hes1 has a critical role in maintenance of intestinal homeostasis by controlling microbiota colonization.

\section{RESULTS}

\section{Hes1 prevents dysbiosis in gut}

As Hes1 global deficiency in mice leads to perinatal lethality, ${ }^{15}$ to investigate the in vivo functions of Hes1 in gut in adult mice, we generated an inducible tamoxifen-dependent Hes1 knockout mouse model (Hes $1^{f l f l}$ Cre-ERT2, designated as Hes1-KO hereinafter). Furthermore, to study the potential long-term effects of constitutive deletion of Hes1 in IECs, we also generated mice lacking Hes1 specifically in IECs (Hes f $^{\text {llfl }}$ Villin-Cre, designated as Hes $1^{\mathrm{IEC}-\mathrm{KO}}$ hereinafter). The deletion efficiency of Hes1 was shown at the protein level by immunohistochemistry and at the mRNA level by quantitative PCR (qPCR) analysis (Supplementary Figure 1 online). We then assessed the impact of Hes1 deficiency on the abundance of total commensal bacteria and several specific bacteria groups (Bacteroidetes phylum and Firmicutes phylum) in the contents of ileum and colon by qPCR. The total bacterial numbers were decreased by 10-folds in ileum of Hes1-KO mice compared to control littermates (Figure 1a). In particular, Hes1-KO mice showed reduction of the numbers of Bacteroidetes phylum by over 1,000 times and of Eubacterium rectale by $\sim 10$ times in the ileum (Figure 1a). However, these changes were not observed in the colon (Figure 1b). In contrast, Hes1-KO mice showed increased Lactobacillus numbers by $\sim 10$ times in the colon (Figure 1b). Hes $1^{\mathrm{IEC}-\mathrm{KO}}$ mice also showed decreased total bacterial numbers and Bacteroidetes numbers in ileum but not in the colon (Figures $\mathbf{1 c}$ and $\mathbf{d}$ ), implicating an IEC-intrinsic role of Hes 1 in regulating commensal bacterial colonization. To exclude the possibility that certain environment factors may influence the microbiota ecology, cohoused Hes1-KO littermates were monitored for bacterial composition post tamoxifen-induced deletion. Results showed that Bacteroidetes from these mice were gradually depleted along with Hes 1 deletion, whereas neither Hes3 nor Hes5 levels were decreased (Figure 1e), supporting the notion that Hes1 deletion was causally correlated with the changes in microbiota. Analysis of Bacteroidetes levels in each group of these littermates also showed the similar results (Supplementary Figure 2a). Next, $16 \mathrm{~S}$ rDNA sequencing was used to comprehensively analyze the bacterial compositions in the Hes1-KO ileum and colon. The sequencing results confirmed that the abundance of S24-7 family from Bacteroidetes phylum was depleted in Hes1-KO ileum (Figures 1f-h). In addition, the weighted UniFracprincipal coordinates analysis analyses showed that the microbiome in Hes1-KO ileum clustered separately from that in control ileum (Figure 1i). 16S rDNA sequencing of colon showed that the abundance of Verrucomicrobiaceae family was increased in Hes1-KO colon (Figures 1j-1). The weighted UniFrac-principal coordinates analysis analyses also showed that the microbiome in Hes1-KO colon clustered separately from that in control colon (Figure 1m). We further applied linear discriminant analysis effect size analysis to the microbiome data and found several differentially abundant clades in control and Hes1-KO mice (Supplementary Figures 3 and 4). For example, in Hes1KO mice, a reduction in Bacteroidetes (including S24-7 family) and Allobaculum, and an increase in E. coli, Buchnera, and Turicibacter genera were observed (Supplementary Figure 3). Then, we used qPCR to confirm that the abundance of E. coli was increased in both the ileum and colon from Hes1-KO as well as from $\mathrm{Hes}^{\mathrm{IEC}-\mathrm{KO}}$ mice (Figures 1n and o). Furthermore, we also found that A. muciniphila, a species of intestinal mucin-degrading bacterium, was highly enriched in colon but not ileum in both Hes1-KO and Hes $1^{\mathrm{IEC}-\mathrm{KO}}$ mice relative to control mice (Figures $\mathbf{1} \mathbf{p}$ and $\mathbf{q}$ ). Moreover, we further compared the fecal microbiota before and after Hes1 deletion in the same Hes $1^{f l / f l}$ Cre-ERT2 mice and observed similar differential regulation of A. muciniphila and E. coli by Hes1 (Supplementary Figure 2b). In summary, these findings reveal that Hes1 deficiency in gut epithelia results in dysbiosis in both small intestine and colon.

\section{Hes1 regulates the expression of AMPs in a microbiota- dependent manner}

Given that the expression and secretion of many intestinal AMPs can be regulated by microbiota, ${ }^{2}$ we next examined the effects of Hes1 deletion on the expression of AMP genes in the ileum and colon. In adult Hes1-KO mice, the RNA levels of Saal, Reg3b, and Reg3g were diminished in the ileum but not in the colon (Figures 2a-c), and the protein level of Reg3g was also reduced in Hes1-KO mice compared with controls (Figure 2d). However, the expression of Reg3 genes were not changed in Hes $1{ }^{\text {IEC-KO }}$ ileum (Supplementary Figure 3), which might be explained by the fact that the extent of Bacteroidetes depletion was more apparent in Hes1-KO ileum than in Hes $1^{\mathrm{IEC}-\mathrm{KO}}$ ileum (Figures 1a and $\mathbf{c}$ ). To test whether the regulation of AMP genes by Hes1 was microbiota-dependent, we treated control and Hes1-KO mice with a mixture of broad-spectrum 

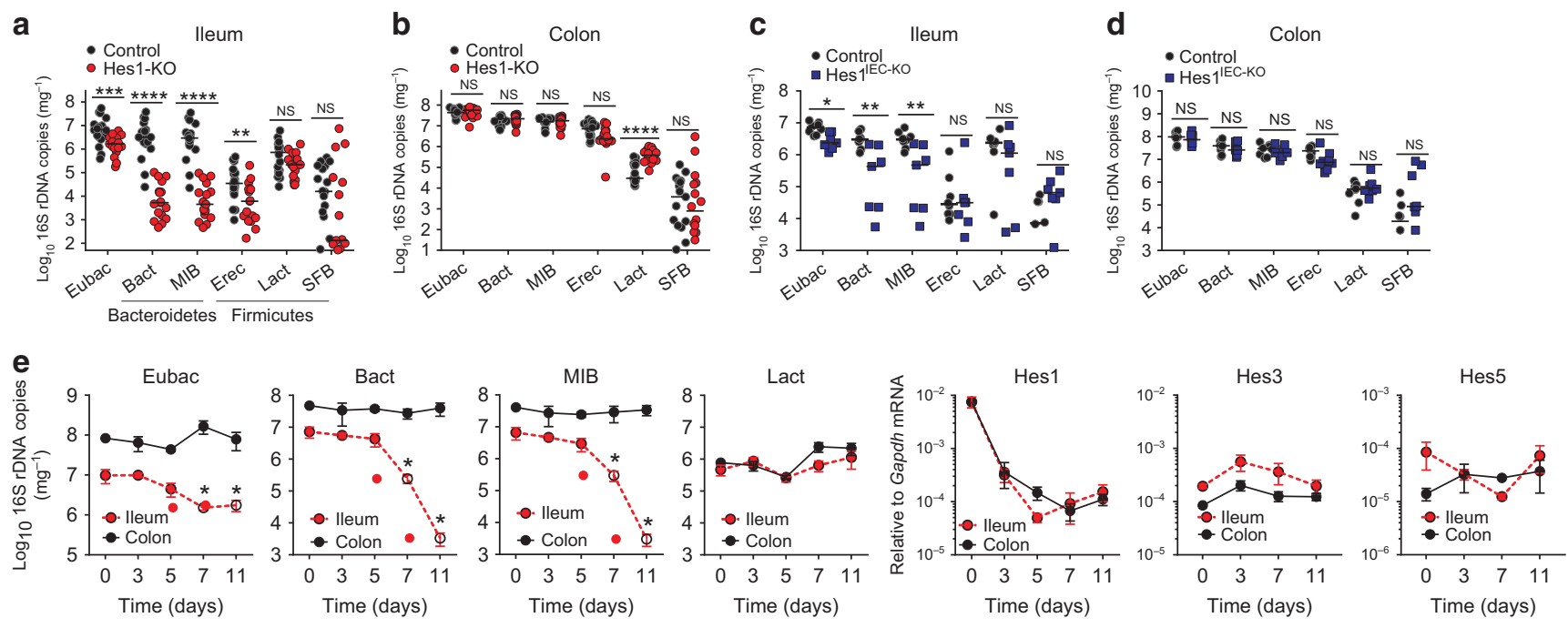

Tamoxifen treatment $\left(H e s 1^{\text {fl/fl }}\right.$ Cre-ERT2)

f
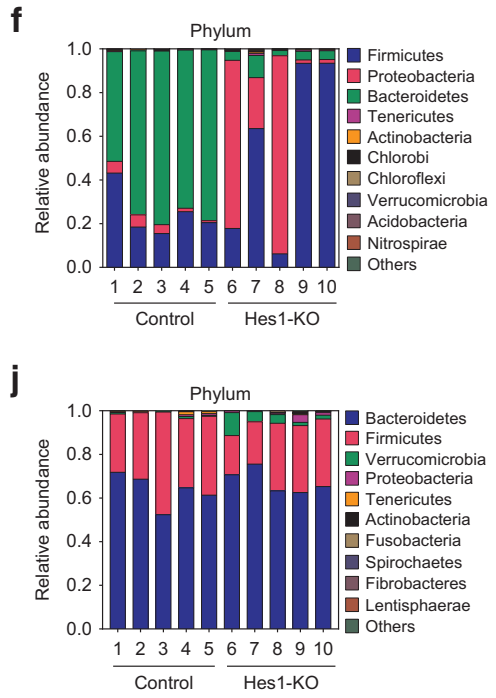

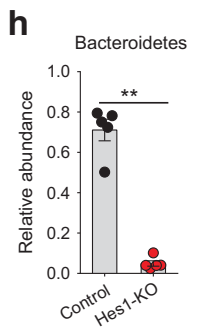

k

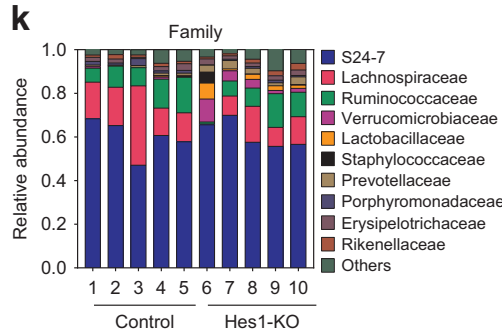

I

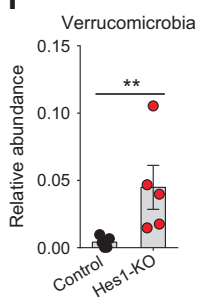

i

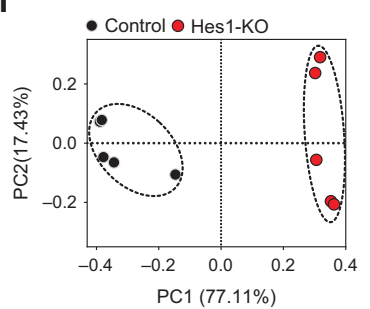

m

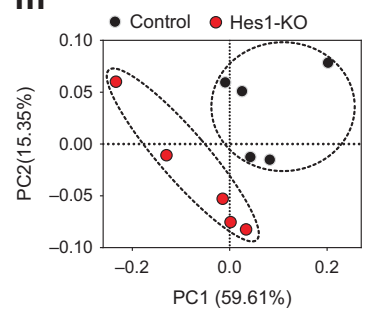

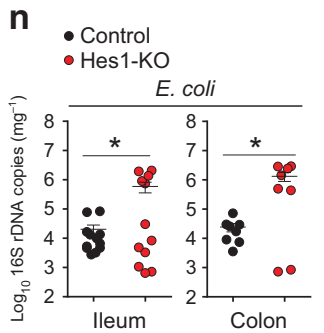
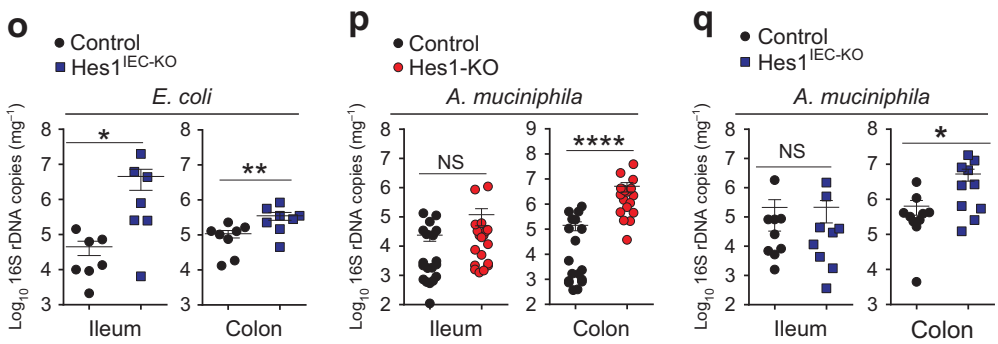

Figure 1 Hairy and enhancer of split 1 (Hes1) deficiency results in dysbiosis in gut. (a,b) Quantitative PCR (qPCR) analysis of specific bacterial 16S rDNA in the ileum (a) and colon (b) of littermate control and Hes $1^{f / / f l}$ Cre-ERT2 (Hes1-KO) mice after 11 days of tamoxifen treatment (a), control, $n=18$; Hes1-KO, $n=17$; (b) control, $n=17$; Hes1-KO, $n=16$ ). (c,d) Analysis of specific bacterial 16S rDNA in the ileum (c) and colon (d) of Hes ${ }^{f / / f /}$ Villin-Cre $\left(\right.$ Hes $\left.1^{\mathrm{IEC}-\mathrm{kO}}\right)$ mice $(n=7)$ and littermate controls $(n=8)$. (e) Analysis of specific bacterial 16S rDNA and Hes $1 / 3 / 5$ mRNA expression in the ileum and colon of co-housed littermates of Hes $1^{f / / f l}$ Cre-ERT2 mice at the indicated time points after tamoxifen treatment $(n=6$ for day $0,3,7,11 ; n=3$ for day 5$)$. $(\mathbf{f}, \mathbf{g})$ lleum contents of control and Hes1-KO mice 11 days post tamoxifen treatment were analyzed for commensal diversity at the phylum level (f) and family level (g) by 16S rDNA sequencing. (h) Abundance of ileum Bacteroidetes is shown for mice with given genotypes. (i) Principal coordinate analysis (PCoA) of weighted UniFrac distances based on ileum $16 \mathrm{~S}$ rDNA analysis of microbiota ( $\mathbf{f}-\mathbf{i}), n=5$ per group). (j,k) Colon contents of control and Hes1KO mice 11 days post tamoxifen treatment were analyzed for commensal diversity at the phylum level (j) and family level (k) by $16 \mathrm{~S}$ rDNA sequencing. (I) Abundance of colon Verrucomicrobia is shown for mice with given genotypes. (m) PCoA of weighted UniFrac distances based on colon 16S rDNA analysis of microbiota $(\mathbf{j}-\mathbf{m}), n=5$ per group). ( $\mathbf{n}, \mathbf{0})$ Analysis of $E$. coli $16 \mathrm{~S}$ rDNA in the ileum and colon of Hes $1-\mathrm{KO}(\mathbf{n})$ and Hes $1^{\mathrm{IEC}-\mathrm{KO}}$ mice $(\mathbf{o}, \mathbf{n}), n=8$ 12 per group; (o), $n=7-8$ per group). (p,q) Analysis of $A$. muciniphila $16 \mathrm{~S}$ rDNA in the ileum and colon of Hes $1-\mathrm{KO}(\mathbf{p})$ and Hes $1^{1 \mathrm{EC}-\mathrm{KO}}$ mice $(\mathbf{q}, \mathbf{p}) n=19$ per group; (q) $n=10$ per group). Data are pooled from three to seven independent experiments (a,b,e,n-q) or three independent experiments (c,d). Data are averages \pm s.e.m.; ${ }^{\star} P<0.05$; ${ }^{\star \star} P<0.005 ;{ }^{* \star} P<0.0005$; ${ }^{* \star \star *} P<0.0001$; NS, not significant (Mann-Whitney test). Horizontal lines (a-d) represent the median values. 

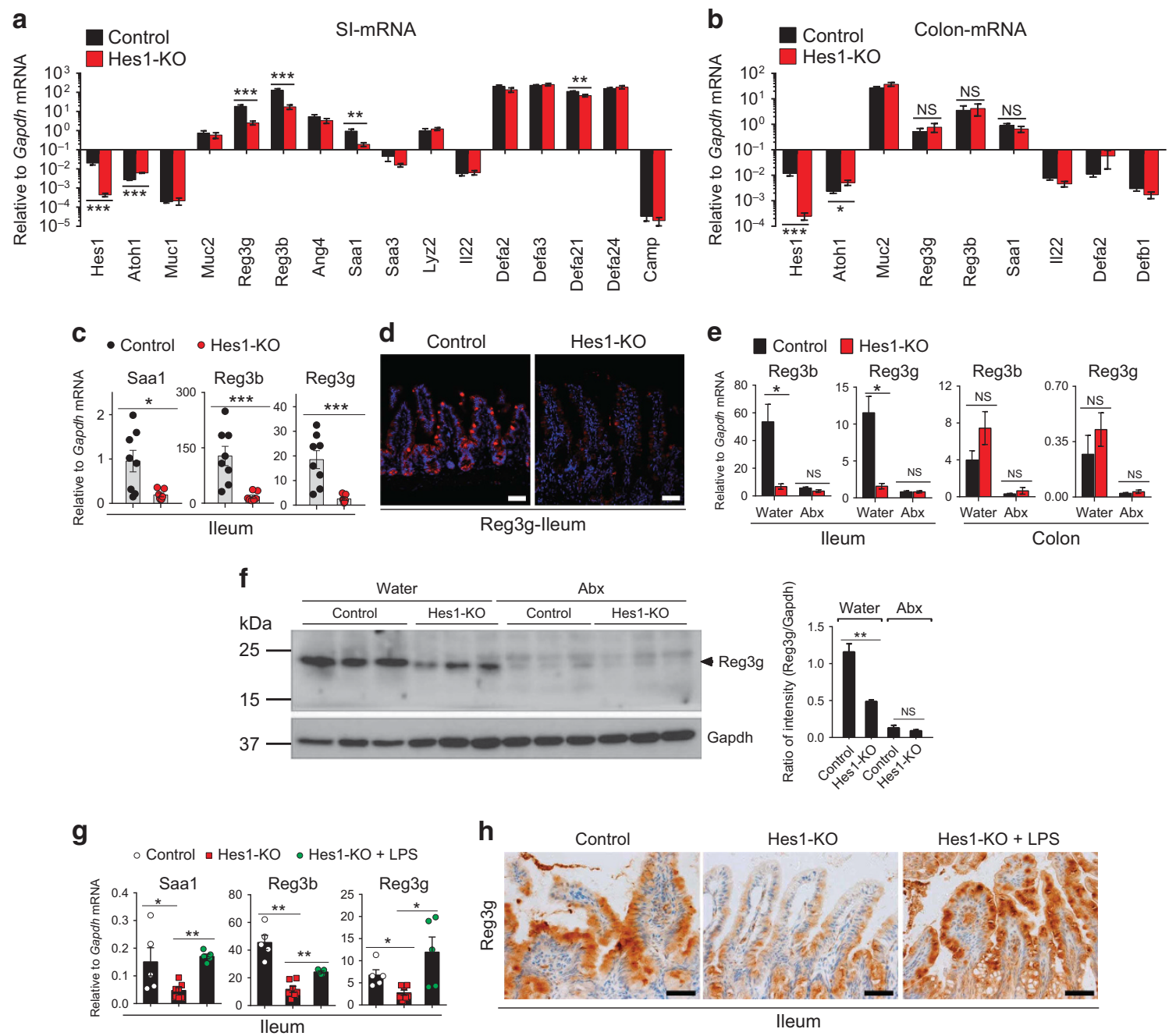

Figure 2 Hairy and enhancer of split 1 (Hes1) regulates antimicrobial gene expression in small intestine in a microbiota-dependent manner. (a,b) Analysis of the mRNA expression in the ileum (a) and colon (b) of Hes1-KO mice and control (a, b), control, $n=6$; Hes1-KO, $n=6$ ). (c) mRNA levels of the indicated AMPs (Saa1, Reg3b, and Reg3g) in control and Hes1-KO ileum (control, $n=8$; Hes1-KO, $n=7$ ). (d) Fluorescent immunohistochemistry analysis of Reg $3 \mathrm{~g}$ protein levels ( $n=3$ per group). (e) mRNA levels of Reg3b and Reg3g in the ileum and colon of control and Hes1-KO mice with or without broad-spectrum antibiotics (Abx) treatment ( $n=3$ per group). (f) Western blot analysis and quantification (right) of Reg3g protein levels in the ileum of mice as in e. Each lane represents an individual mouse. (g.h) Hes1-KO mice were orally administered with vehicle or 2 mg of lipopolysaccharides (LPS). RNA and tissue samples were collected $48 \mathrm{~h}$ later. (g) mRNA levels of the indicated antimicrobial proteins (AMPs) (Saa 1, Reg3b, and Reg3g) in the ileum (control, $n=5$; Hes1-KO, $n=7$, Hes1-KO + LPS, $n=5$ ). (h) Immunohistochemical analysis of Reg3g protein levels in mice as in i. Scale bars, $50 \mu \mathrm{m}$. ${ }^{\star} P<0.05 ;{ }^{\star \star} P<0.005 ;{ }^{\star \star \star} P<0.0005$; NS, not significant (Mann-Whitney test). Data are averages \pm s.e.m.; data are representative of three independent experiments.

antibiotics, to deplete their microbiota. Upon antibiotics treatment, control mice exhibited diminished AMP (Reg3b and $R e g 3 g$ ) mRNA and protein levels in the ileum, comparable to those observed in Hes1-KO mice (Figures 2e and f), suggesting that Hes1-mediated regulation of AMPs might be microbiota-dependent. We next aimed to assess whether loss of microbiota was responsible for diminished AMP expression in Hes1-KO mice by reconstituting microbe-dependent signaling via lipopolysaccharides (LPS) treatment in vivo. Oral administration of LPS did restored the expression of AMPs in the ileum of Hes1-KO mice at both mRNA and protein levels (Figures $2 \mathbf{g}$ and $\mathbf{h}$ ). Collectively, these data suggest that Hes1 regulates intestinal antimicrobial gene expression by controlling microbiota colonization.

\section{Hes1 promotes resistance against pathogenic bacterial colonization}

Studies have revealed that commensal microbiota contributes to colonization resistance against intestinal pathogens. ${ }^{25}$ Thus, we next tested whether dysbiosis caused by Hes1 deficiency would influence the colonization of pathogenic bacteria. At the early stage of infection with Citrobacter rodentium, Hes1-KO mice exhibited higher bacterial burdens in the ileum, cecum, and colon than control mice (Figures 3a-d). Then we tested another pathogenic bacterial species, E. coli O157:H7, which also colonized more efficiently in the ileum and colon of Hes1KO mice compared with control mice (Figure 3e). Thus, these results show that Hes1-deficent mice are highly susceptible to intestinal pathogenic bacterial colonization. 


\section{Hes1 deficiency promotes inflammation in gut}

As dysbiosis is often associated with disturbed gut homeostasis, we then assessed whether Hes1 deficiency would alter the status of intestinal inflammation. DSS (2.0-3.0\%) was used to induce inflammation in mice with the clinical and histological features that partially resemble inflammatory bowel disease. Compared with control mice, Hes1-KO mice that were subjected to DSS treatment exhibited profound weight loss and increased disease severity with high mortality (Figures $\mathbf{3 f}$ and g). To elucidate whether this phenotype was intrinsic to epithelial cells, Hes $1^{\text {IEC-KO }}$ mice were subjected to low dose (2\%) DSS administration. Hes $1^{\text {IEC-KO }}$ mice also showed profound weight loss, enhanced disease severity, loss of crypt architecture, and extensive intestinal ulceration in the ileum and colon, whereas control mice were minimally affected (Figures $3 \mathrm{~h}$ and i), supporting the notion that epithelium-intrinsic Hes1 alleviated inflammation in the DSS model. Studies have shown that Hes1 modulates cell proliferation in multiple organs ${ }^{14}$. We then asked whether Hes1 deficiency possibly impaired IEC proliferation and thus compromised epithelial integrity. The histological analyses revealed that intestinal architecture appeared dilated in Hes1-deficient ileum (Supplementary Figure 4a), and that Hes1-deficient ileum was even more proliferative with deeper crypts than control ileum (Supplementary Figure $4 \mathbf{b}$ ). Thus, the susceptibility to inflammation in Hes1-deficient mice might not be due to a

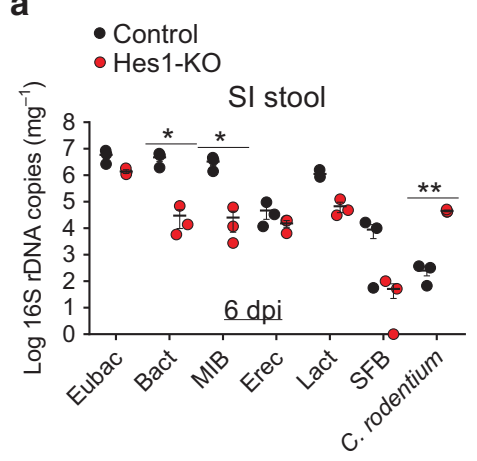

e Control

- Hes1-KO

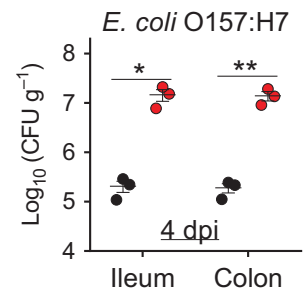

b

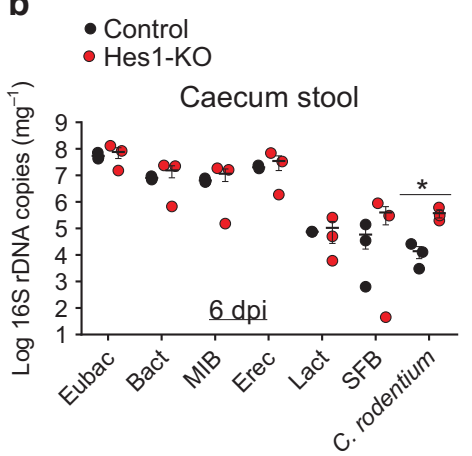

C

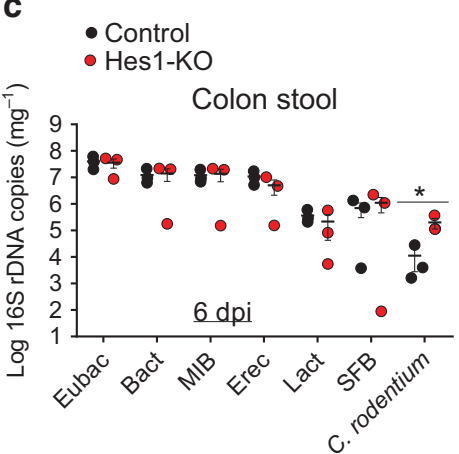

d $\quad$ Control

- Hes1-KO

Fecal

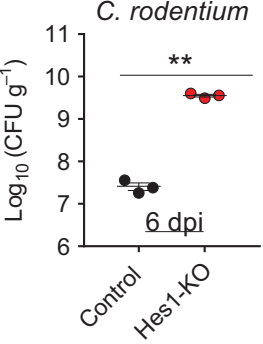

f
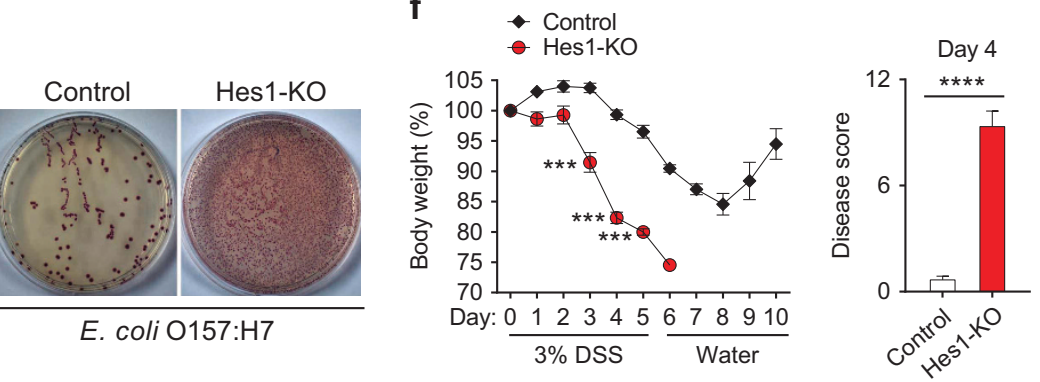

g

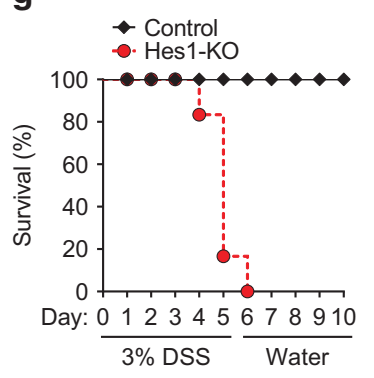

h

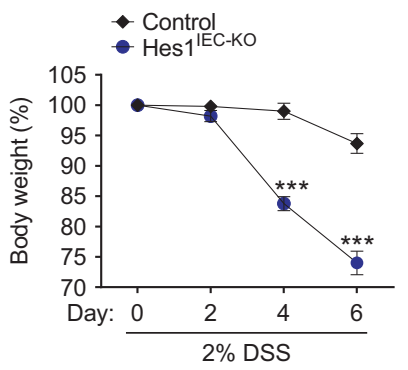

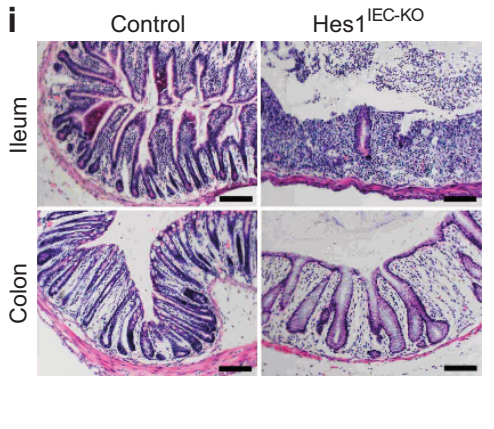

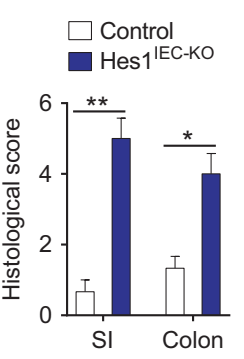

Figure 3 Hairy and enhancer of split 1 (Hes1) deficiency enhances susceptibility to pathogenic bacteria colonization and promotes inflammation in gut. (a-c) Analysis of specific bacterial 16S rDNA in the ileum (a), cecum (b), and colon (c) of control and Hes1-KO mice ( $n=3$ per group) 6 days post $C$. rodentium infection. (d) Bacterial burdens of $C$. rodentium in feces 6 days post infection. (e) Bacterial colony-forming units (CFUs) of $E$. coliO157:H7 in the ileum and colon of control and Hes1-KO mice ( $n=3$ per group) 4 days post $E$. coli O157:H7 infection. Colon content samples collected at day 4 post infection were resuspended in phosphate buffer saline (PBS) and added to chromogenic media agar plates for single colony visualization (right). (f) Body weight measurements and histological scores of Dextran sodium sulphate (DSS)-treated control and Hes1-KO mice at day 4 ( $n=6$ per group). (g) Left panel shows survival curves of mice treated as in $\mathbf{f}$ and the right panel shows bloodstain in the padding of cages. (h) Body weight measurements and disease scores of DSS-treated control and Hes $1^{1 \mathrm{EC}-\mathrm{KO}}$ mice at day 6 (control, $n=6$; Hes $1^{\mathrm{IEC}-\mathrm{KO}}, n=3$ ). (i) Mucosal histology of DSS-challenged mice at day 6 was scored based on hairy and enhancer of split 1 (H\&E) staining. Scale bars, $100 \mu \mathrm{m}$. Data are averages \pm s.e.m.; ${ }^{*} P<0.05$; ${ }^{\star \star} P<0.005$; ${ }^{* \star \star \star} P<0.0001$ (Mann-Whitney test). Data are representative of three independent experiments. 


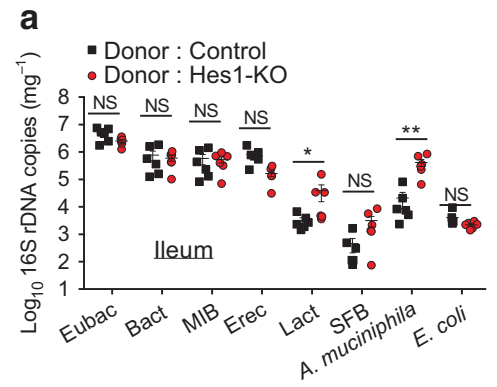

C

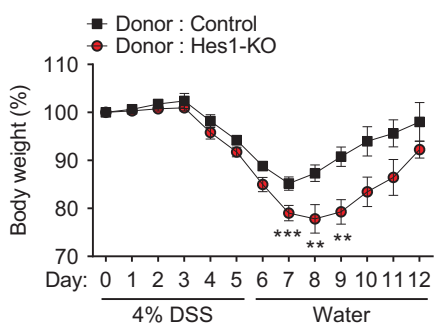

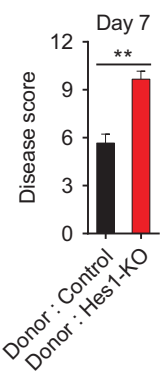

b

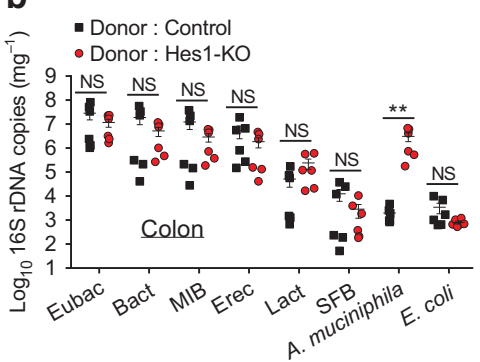

d

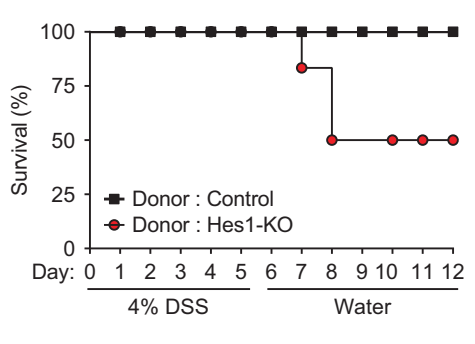

e

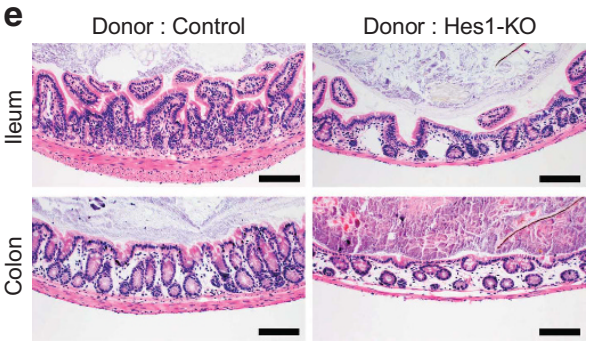

Figure 4 Hairy and enhancer of split 1 (Hes1)-deficient-mouse-derived fecal microbiota promotes intestinal inflammation. (a,b) Analysis of specific bacterial $16 \mathrm{~S}$ rDNA of small intestine (a) and colon (b) microbiota in control recipient mice that received fecal microbiota transplantation from control mice or Hes1-KO mice. (c) Body weight measurements at the indicated time points and disease scores of at day 7 post DSS treatment of recipient mice (donor: control; donor: Hes1-KO). (d) Survival curves of mice treated as in c. Number of mice per group in a-d: $n=6$ per group. (d) Mucosal histology of the above DSS-challenged mice at day 12 was analyzed by hairy and enhancer of split 1 (H\&E) staining. Scale bars, $100 \mu \mathrm{m}$. Data are averages \pm s.e.m. ${ }^{* \star} P<0.005 ;{ }^{* \star \star} P<0.0005$ (Mann-Whitney test). Data are pooled from two-three independent experiments.

defective epithelial architecture. Taken together, these findings show that Hes1 deficiency promotes inflammation in small intestine and colon.

\section{Hes1-deficient-mouse-derived fecal microbiota promotes intestinal inflammation}

To further corroborate the notion that dysbiosis contributed to increased intestinal inflammation in Hes1-deficient mice, we performed fecal-microbiota transplantation experiments by transferring the microbiota contents from Hes1-KO mice or control littermates into antibiotic-pretreated control mice. Five days post fecal transplantation, we detected higher colonization of A. muciniphila in mice that received Hes1-KO microbiota than that in mice receiving control microbiota (Figures $4 a$ and b). Notably, upon DSS challenge, Hes1-KO microbiota recipients exhibited more profound weight loss, greater disease severity, and higher mortality rate than control microbiota recipients (Figures $4 \mathbf{c}$ and d). Compared with control recipients, the mice that received Hes1-KO microbiota also displayed signs of destructive tissue damage and showed loss of crypt architecture in both the ileum and colon after DSS treatment (Figure 4e). In summary, these findings demonstrate that Hes1-deficiency-induced dysbiosis increases host susceptibility to intestinal inflammation.

Hes1 deficiency induces mucus hyperplasia in adult mice Studies have revealed that Notch signaling controls the fate of IECs in embryonic gut by activating the expression of Hes family genes. ${ }^{15,16,19}$ However, due to the potential redundancy among multiple Hes family members, whether Hes1 alone plays a pivotal role in regulating the fate of IECs in adult mice is still unclear. We then characterized the differentiation statuses of IECs in Hes1-KO mice and Hes $1^{\text {IEC-KO }}$ mice. The histological and immunohistochemical analysis showed that the numbers of Paneth cells (lysozyme ${ }^{+}$) and goblet cells (Periodic acidSchiff $^{+}$and $\mathrm{Muc}^{+}$) were increased in the small intestine of Hes1-KO mice and Hes $1^{\mathrm{IEC}-\mathrm{KO}}$ mice (Figures 5a-c). Likewise, the numbers of goblet cells were also increased in the colon of Hes1-KO mice and Hes1 $1^{\mathrm{IEC}-\mathrm{KO}}$ mice (Figures 5d and e). Importantly, the crypts were filled with increased amount of mucus in both Hes1-KO and Hes $1^{\mathrm{IEC}-\mathrm{KO}}$ intestine (Figures 5ad). Thus, these findings indicate that Hes1 deficiency induces mucus hyperplasia in the intestine of adult mice.

\section{Hes1 regulates microbiota ecology partially through changing mucosal microhabitats}

Emerging evidence suggests that the mucus layers of intestinal tract provide protective microhabitats for commensal bacteria colonization that may serve as reservoirs for microbiota that can be re-seeded into the gut lumen. ${ }^{10}$ Notably, Hes1 deficiency is associated with goblet cell hyperplasia and mucus overproduction. Thus, we hypothesized that Hes1 might regulate microbiota ecology by changing mucus-associated mucosal microhabitats. To test our hypothesis, we firstly investigated whether Hes1 regulated microbiota colonization in gut mucosal microhabitats by probing microbiota compositions within the mucus layers. The results demonstrated that there were less Bacteroidetes in the small intestinal mucosal microhabitats in Hes1-KO mice than in controls (Figure 5f), whereas Hes1-KO colonic mucosal microhabitats harbored more Lactobacillus and A. muciniphila than control mice (Figure 5g). Such 

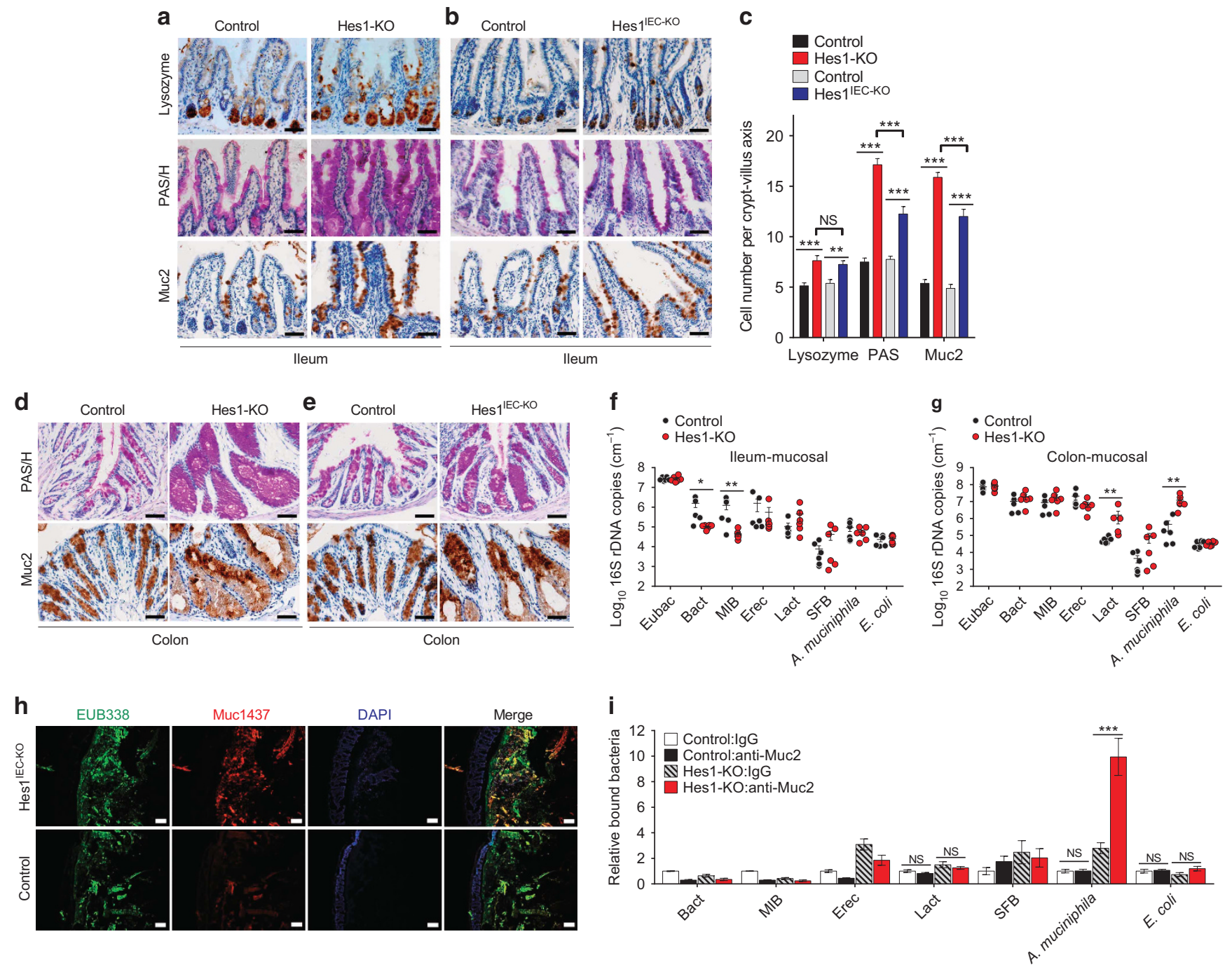

Figure 5 Hairy and enhancer of split 1 (Hes)1 regulates microbiota ecology partially through altering mucosal microhabitats. (a-c) Immunohistochemistry illustrating the small intestinal epithelia of control, Hes1-KO (a) and Hes $1^{\text {IEC-KO }}$ (b) mice. Lysozyme, Periodic acid-Schiff (PAS), and Muc2 staining labels Paneth cells, goblet cells, and all the Muc2-positive intestinal epithelial cells (IECs), respectively. (c) Positive cells were enumerated in each crypt-villus units. (d,e) PAS and Muc2 staining in the colon of control, Hes1-KO (d) and Hes $1^{1 \mathrm{EC}-\mathrm{KO}}$ (e) mice. Control animals corresponded to littermate controls of mice with given genotypes. Scale bars, $50 \mu \mathrm{m}$. (a-e) $n=3$ per group). (f,g) Analysis of specific bacterial $16 S$ rDNA of mucosal bacteria in the ileum (f) and colon ( $\mathbf{g}$ ) of control and Hes1-KO mice ( $n=6$ per group). (h) Fluorescence in situ hybridization (FISH) using EUB338 probe for all bacteria (green), Muc1437 probe for A. muciniphila (red), and 4',6-diamidino-2-phenylindole (DAPI) (blue) in the Carnoy's fixed colon from control and Hes $1^{1 \mathrm{EC}-\mathrm{KO}}$ mice. Scale bars, $100 \mu \mathrm{m}$. (i) Quantitative PCR of Muc2-bound and unbound bacteria in the colon of control and Hes1-KO mice. Results represented the relative enrichment of DNA amounts of Muc2-bound specific bacteria versus total bacteria and were normalized to DNA content in control IgG-bound bacteria that was set as 1 ( $n=6$ per group). Data are averages \pm s.e.m.; ${ }^{*} P<0.05$; ${ }^{\star \star} P<0.005 ;{ }^{* \star *} P<0.0005$; NS, not significant (MannWhitney test). Data are representative of three independent experiments.

alterations of mucosal microbiota were consistent with lumen microbiota changes in Hes1-KO mice. We then examined the spatial distribution of bacteria adjacent to the mucosa in the colon by fluorescence in situ hybridization (FISH) using a universal bacterial $16 \mathrm{~S}$ ribosomal RNA probe and a $A$. muciniphila-specific 16S rRNA probe. Consistent with qPCR data shown in Figure 5g, the FISH assays showed enhanced A. muciniphila colonization in the mucosal microhabitats in Hes1 ${ }^{\mathrm{IEC}-\mathrm{KO}}$ colon compared to control colon (Figure 5h). As mucus overproduction was observed in the intestinal tract of Hes1-KO mice, we speculated that aberrant mucus microenvironment might be partially responsible for dysbiosis in Hes1-KO mice. To test our hypothesis, we detected the mucus-associated bacteria in colon by performing Muc2-pulldown experiments. Our data showed that A. muciniphila was substantially enriched in Muc2-bound bacteria in Hes1-KO fecal suspensions (Figure 5i), indicating that mucus preferentially associated with A. muciniphila in Hes1-KO mice and that enrichment of $A$. muciniphila in colonic lumen of Hes1-deficient mice may stem from changes in mucosal microhabitats. As Notch inhibition also induce mucus hyperplasia ${ }^{17}$, we next treated C57BL/6J mice with the Notch inhibitor dibenzazepine to see whether Notchinhibition-induced mucus hyperplasia induces such dysbiosis. Unexpectedly, dibenzazepine treatment did not influence fecal microbiota composition (Supplementary Figure 7), suggesting 
that Hes1-deficiency-induced dysbiosis did not result from mucus hyperplasia alone. Probably, Hes1 deficiency upregulated some unknown factors to contribute the unbalanced mucosal microhabitats. Thus, these findings suggest that Hes1 regulates microbiota ecology partially through changing mucosal microhabitats. Taken together, our study unravels a role of epithelial Hes1 in maintaining intestinal homeostasis by preventing dysbiosis.

\section{DISCUSSION}

Intestinal homeostasis is coordinately maintained by the gut epithelium, mucosal immune cells, and commensal microbiota. In this study, we found that epithelial Hes1 deficiency led to intestinal dysbiosis and subsequently increased susceptibility to pathogen infections and intestinal inflammation. Unbalanced mucosal microhabitats was partially responsible for the dysbiosis. Both methods of Hes1 deletion caused increase of E. coli in ileum and colon, and increase of A. muciniphila in colon, suggesting a IEC-intrinsic regulation of $E$. coli and $A$. muciniphila by Hes1. However, inducible Hes1 deletion (Hes1$\mathrm{KO}$ ) resulted in loss of Bacteroidetes in the ileum much more apparently than IEC-intrinsic Hes1 deletion $\left(\mathrm{Hes}^{\mathrm{IEC}-\mathrm{KO}}\right)$, which was consistent with the expression patterns of microbiota-dependent AMPs in these mice. These data suggest that Hes1 expression in cells other than IECs may be also involved in the regulation of Bacteroidetes abundance in ileum. In fact, immune cells can regulate microbiota ecology through directly secreting proteins such as $\operatorname{IgA}^{26}$ or indirectly regulating IEC production of AMPs via cytokines such as IL-17 or IL-22. ${ }^{2}$ In our model, inducible Hes1 deletion could also decrease the expression of Hes1 in cell types such as macrophages ${ }^{22}$ and lymphocytes. Of note, it has been reported that Hes1 regulates the function and differentiation of these immune cells. ${ }^{23,27}$ Thus, the regulation of immune cells by inducible Hes1 deletion may also contribute to the loss of Bacteroidetes in the ileum. Whether Hes1 in these immune cells is involved in the regulation of microbiota remains to be elucidated in future studies.

Intestinal epithelial AMPs have an essential role in allowing epithelial surfaces to cope with surrounding microorganisms. Paneth cells reside at the base of the crypts in small intestine, secreting large amounts of AMPs that limit the growth of bacteria. Our data show that conditional inducible Hes1 deletion resulted in loss of total bacteria numbers in the ileum, which subsequently decreased the expression of Reg $3 \mathrm{~b} / \mathrm{g}$. Loss of Bacteroidetes could not be explained by over production of certain AMPs as Hes1 deficiency did not lead to increased expression of AMP genes such as Reg3b/g, Saa1, Defa2, Defa3, Defa21, and Defa24. However, we could not rule out the possibility that some unknown AMPs may be upregulated under the Hes1-deficient condition to inhibit growth of Bacteroidetes. Instead, we observed goblet cell hyperplasia in small intestine and colon of Hes1-deficient mice. Goblet cells are specialized epithelial cells distributed throughout the gastrointestinal tract that secrete large amounts of gel-forming mucins. A number of studies have shown that microbiota residing in the mammalian intestine are adept at foraging glycans and polysaccharides including those in host mucus ( $O$ linked glycans) ${ }^{28,29}$ Mucin 2 is coated with over 100 different $O$-linked glycans in humans. ${ }^{30}$ These glycans differ among mammals, and the differences in the complex glycans have been proposed to contribute to selective colonization by various microbiota species via their ability to utilize specific glycans. ${ }^{10}$ A. muciniphila, a symbiont in many mammals, is effective at degrading mucin and often found at high abundance in the mucus layer. ${ }^{31}$ Hes1-deficient mice showed higher abundance of A. muciniphila in the colonic lumen and mucosa than control mice and this enrichment of colonization was strongly associated with mucus hyperplasia, indicating that Hes1deficient mice may provide more mucus as nutrition or niches for A. muciniphila in colon. Notably, Bacteroidetes started to decrease after 7 days of tamoxifen treatment in $\mathrm{Hes}^{\mathrm{fl} / \mathrm{fl} \mathrm{Cre}}{ }^{\mathrm{f}}$ ERT2 mice, whereas Hes1 was dramatically decreased after 3 days of tamoxifen treatment (Figure 1e), further supporting the hypothesis that dysbiosis appears after changes in the epithelium itself such as goblet cell hyperplasia. Interestingly, studies have shown that the ability of a bacterium species to utilize a specific and limited nutrient may determine its ability to colonize the gut. ${ }^{10}$ For example, bacterial species-specific carbohydrate utilization systems are identified as commensal colonization factors in Bacteroides fragilis and Bacteroides vulgatus, and the commensal colonization factors are important for these bacteria to colonize saturated nutrient microhabitats. ${ }^{32}$ In addition, our data showed that dibenzazepineinduced mucus hyperplasia alone could not result in such dysbiosis(Supplementary Figure 7). Thus, it will be of interest to investigate whether altered nutritional conditions or some unknown factors in these unbalanced microhabitats cause dysbiosis in the gut of Hes1-deficient mice in future studies.

Microbiota compositions are critical for maintaining intestinal homeostasis ${ }^{5}$. It has been reported that commensal microbiota is required for protection of host from intestinal epithelial injury. ${ }^{33}$ The mice that received combination of antibiotics showed severe mortality and morbidity when given DSS, whereas oral LPS administration rescued the commensal depleted mice from DSS-induced mortality. However, oral LPS did not protect the commensal depleted TLR4-deficient mice from DSS challenge. ${ }^{33}$ The data suggest that recognition of commensal microbes by TLRs is critical for protecting host from intestinal epithelial damage. Hes1 deficiency clearly induced dysbiosis such as loss of Bacteroidetes in the ileum (Figure 1) and pathogenic changes were also observed in the ileum of Hes $1^{\mathrm{IEC}-\mathrm{KO}}$ mice in the DSS-induced colitis model (Figure 3i). Thus, we speculate that dysbiosis may dispose Hes1-deficient ileal epithelial cells hypersensitive to the DSS challenge. However, we could not totally exclude the possibility that intrinsic alterations of epithelial cells may also be associated with pathogenic changes in the ileum of the Hes1 KO mice subjected to the colitis model.

Goblet cell depletion has been regarded as a characteristic associated with mucosal inflammation and colon cancer ${ }^{3}$. As mucin 2 , the principal secretory product, is a key component of 
the mucus layer that protects the mucosal surfaces from microbial challenges ${ }^{4}$. Muc2 deficiency results in direct contact between microorganisms and the epithelial barrier, promoting intestinal inflammation and cancer development in mice ${ }^{4}$. Similarly, it has been reported that decreased goblet cell number by claudin-1-mediated Notch activation enhances susceptibility to mucosal inflammation ${ }^{34}$. In contrast, mucus hypersecretion upon Pofut1 inactivation, which mediates inactivation of Notch signaling, has also been shown to exacerbate inflammation in colitis ${ }^{35}$. Thus, these seemingly conflicting studies elude to a notion that in order to properly maintain mucosal homeostasis, mucus production must be tightly controlled, and that either overproduction or underproduction of mucus can lead to disturbed homeostasis. In line with this thinking, our data regarding Hes1-deficient mice showed that mucus hyperplasia was associated with dysbiosis, leading to disturbed homeostasis and intestinal inflammation.

In summary, our study sheds new light on the role of epithelial Hes1 in maintaining microbiota ecology through modulating mucosal microhabitats to maintain intestinal homeostasis and may provide novel insights into designing therapeutic approaches that target microbiota in inflammatory bowel disease.

\section{METHODS}

Mice. C57BL/6J mice were purchased from Jackson Laboratory (Bar Harbor, ME). Hes $1^{f l f l}$ mice were originally obtained from $\mathrm{R}$ Kageyama $^{36}$. Mice with inducible deletion of Hes1 (Hes $1^{f l f l}$ Cre-ERT2) were generated by crossing Hes $1^{\text {fllfl }}$ mice to Cre-ERT2 mice on the C57BL/6J background. Expression of Cre-ERT2 was under the control of the promoter of Rosa26. Villin-Cre mice were purchased from Jackson Laboratory. Mice with IEC-specific deletion of Hes1 (Hes $1^{f l}$ ${ }^{f l}$ Villin-Cre) were generated by crossing Hes $1^{f l /}+$ Villin-Cre mice with Hes $1^{f l /+}$ Villin-Cre mice, and Hes $1^{+/+}$Villin-Cre littermates were used as controls. Littermates were used for each single independent experiment. All mice were used at $8-12$ weeks of age and littermates were randomly divided into different groups. The littermates were single-housed after weaning unless otherwise specified. No statistical methods were used to predetermine sample sizes. Histological analyses were performed in a blinded manner and the sample sizes represent biological replicates. For Hes $1^{f l f l}$ Cre-ERT2 mice, deletion of Hes1 was induced by intraperitoneal injection with $2 \mathrm{mg}$ per mouse of tamoxifen (dissolved in corn oil) for three times during the course of three days, and Hes $1^{f l / f l}$ Cre-ERT2 littermates without tamoxifen treatment were used as controls. For the inducible deletion of Hes1, mice were used for experiments 11 days post injection unless otherwise specified. These mice were single-housed after tamoxifen treatment unless otherwise specified. All mice were kept under specific pathogen-free (SPF) conditions at the Experimental Animal Facility, Tsinghua University. All animal experiments were reviewed and approved by the Institutional Animal Care and Use Committees at Tsinghua University.

Isolation of bacterial DNA. For isolation of lumen bacterial DNA, the intestinal tract was excised and the distal $5 \mathrm{~cm}$ of the small intestine (ileum) and colon was isolated. The luminal contents were collected by flushing with $1 \mathrm{ml}$ sterile phosphate buffer saline (PBS), weighed, and homogenized. For isolation of mucosal bacterial DNA, the intestines were flushed vigorously, opened up longitudinally, cut into pieces, and then vortexed vigorously in $1 \mathrm{ml}$ sterile PBS to collect the bacteria adherent to the mucosal surface and inner mucus layer. The luminal and mucosal bacterial DNA was immediately isolated with Stool Genomic DNA Kit (CWBIO, Beijing, China) according to the manufacturer' instructions.
qPCR for microbiota analysis. The abundance of specific intestinal bacterial groups was measured by quantitative real-time PCR (qPCR) with FastSYBR mixture (CWBIO) and universal $16 \mathrm{~S}$ rDNA primers ${ }^{37}$ (Supplementary Table S1) on a real-time PCR system (StepOnePlus; Applied Biosystems, Foster City, CA). Bacterial abundance was determined using standard curves with reference to cloned bacterial DNA corresponding to a short segment of the 16S rRNA gene that was amplified using conserved specific primers (Supplementary Table S1). It should be noted that qPCR measures $16 \mathrm{~S}$ rRNA gene copies per sample, not the actual bacterial numbers or colony-forming units.

16S rDNA sequencing and analysis. The bacterial DNA was isolated as described above. 16S rDNA of distinct regions (16S V4) were amplified used specific barcoded primers. All PCR reactions were carried out with Phusion High-Fidelity PCR Master Mix (New England Biolabs, Ipswich, MA). PCR products were purified with Qiagen Gel Extraction Kit (Qiagen, Hilden, Germany). Sequencing libraries were generated using TruSeq DNA PCR-Free Sample Preparation Kit (Illumina, San Diego, CA) following the manufacturer's recommendations and index codes were added. The library quality was assessed on the Qubit@2.0 Fluorometer (Thermo Scientific, Waltham, MA) and Agilent Bioanalyzer 2100 system. At last, the libraries were sequenced on an Illumina HiSeq2500 platform and $250 \mathrm{bp}$ paired-end reads were generated. Paired-end reads were merged using FLASH (V1.2.7, http:// ccb.jhu.edu/software/FLASH/ $/)^{38}$. Quality filtering on the raw tags were performed under specific filtering conditions to obtain the high-quality clean tags according to the QIIME (V1.7.0, http://qiime.org/index.html) ${ }^{39}$. The tags were compared with the reference database (Gold database, http:// drive5.com/uchime/uchime_download.html) using UCHIME algorithm (UCHIME Algorithm, http://www.drive5.com/usearch/manual/uchime_algo.html $)^{40}$ to detect chimera sequences, and then the chimera sequences were removed to obtain the Effective Tags. Sequence analysis was performed by Uparse software (Uparse v7.0.1001, http://drive5.com/ uparse/). Sequences with $\geq 97 \%$ similarity were assigned to the same operational taxonomic units. Representative sequence for each operational taxonomic unit was screened for further annotation. For each representative sequence, the GreenGene Database (http://greengenes.lbl.gov/) was used based on RDP classifier (Version 2.2, http://sourceforge.net/ projects/rdp-classifier/ $)^{41}$ algorithm to annotate taxonomic information. Beta diversity analysis was used to evaluate differences of samples in species complexity. Beta diversity on both weighted and unweighted unifrac were calculated by QIIME software (Version 1.7.0).

Microbiota depletions. The gut microbiota was depleted by feeding mice with broad-spectrum antibiotics in drinking water as previously described ${ }^{33}$. Mice were given ampicillin $\left(1 \mathrm{gl}^{-1}\right.$; Sigma, St. Louis, $\mathrm{MO}$ ), vancomycin (500 $\mathrm{mgl}^{-1}$; Amresco, Solon, OH), neomycin sulphate $\left(1 \mathrm{gl}^{-1}\right.$; Sigma), and metronidazole ( $\mathrm{gl}^{-1}$; Sigma) in drinking water for 5-7 days. For some experiments, mice were given vancomycin (500 $\mathrm{mg} \mathrm{l}^{-1}$; Amresco), streptomycin ( $1 \mathrm{gl}^{-1}$; Amresco), or colistin $\left(1 \mathrm{gl}^{-1}\right.$; Amresco) individually for 7 days.

LPS administration. Animals were depleted of commensal microbiota as described above and then orally administered with LPS purified from E. coli 026:B6 (3 mg per dose; Sigma) by intragastric gavage. After $48 \mathrm{~h}$ of LPS treatment, the animals were killed and the tissues were collected for RNA analysis or fixed with $4 \%$ paraformaldehyde for immunohistochemical analysis.

qPCR for tissue mRNA. Tissues were immediately snap-frozen in liquid nitrogen and homogenized. Total RNA was isolated from homogenized intestinal tissues using Total RNA Purification Kit (GeneMark, Taizhong, China). Complementary DNA was synthesized from $1 \mu \mathrm{g}$ total RNA by M-MLV reverse transcriptase (Takara). Quantitative real-time PCR was performed using FastSYBR mixture (CWBIO) with specific primers (Supplementary Table S1). The comparative threshold cycle method and an internal control (Gapdh) were used to normalize the expression of target genes. 
Western blotting. Tissues were immediately snap-frozen in liquid nitrogen, homogenized, and lysed in RIPA buffer (P0013B; Beyotime, Haimen, China). Equal amounts of protein lysates were run on an SDS-polyacrylamide gel electrophoreiss and transferred onto polyvinylidene difluoride membranes by electro blotting. After blocking with $5 \%$ fat-free milk, the membranes were incubated at $4{ }^{\circ} \mathrm{C}$ overnight with the following primary antibodies: anti-mouse Reg3g (Abgent, Suzhou, China; AP5606c) and Gapdh (Easybio, Beijing, China; $\mathrm{BE0023)}$. The membranes were then washed and incubated with horseradish-peroxidase-conjugated secondary antibody (Cell Signaling, Cambridge, MA). Proteins were visualized with SuperSignal West Pico Chemiluminescent Substrate (Thermo Scientific; 34080).

Histology and immunohistochemistry. Tissues were fixed in $4 \%$ paraformaldehyde, paraffin embedded, and sectioned $(5 \mu \mathrm{m})$. Tissue sections were stained with hematoxylin and eosin, periodic acid-Schiff, anti-Reg3g (Abgent; AP5606c), anti-lysozyme (Santa Cruz, Dallas, TX; sc-27956), anti-Ki67 (Cell Signaling; 12202), and anti-Muc2 (Santa Cruz; sc-15334) for immunohistochemistry, or with anti-Reg3g and 4',6-diamidino-2-phenylindole for immunofluorescence. Slides were washed three times with $0.1 \%$ TBS-Tween before incubation with secondary antibodies. For immunofluorescence, the secondary antibody was conjugated with Alexa Fluor 647 (Abcam, Cambridge, MA). Stained slides were washed again in PBS before 4',6-diamidino-2-phenylindole staining and mounting with SlowFade Diamond Antifade Mountant (Life Technologies, Carlsbad, CA). Similar methods were used for bright-field immunohistochemistry, and signals were developed with DAB (TIANGEN, Beijing, China) in conjunction with a hematoxylin counterstain (Solarbio, Beijing, China). After dehydration, sections were mounted in neutral balsam. All staining assays were repeated in three independent experiments with three mice per group.

Fluorescence in situ hybridization. FISH analysis was performed as previously described ${ }^{42}$. Colon samples were fixed using freshly prepared methanol-Carnoy's fixative (60 methanol, 30 Chloroform, 10\% glacial acetic acid) for $4 \mathrm{~h}$ at $4{ }^{\circ} \mathrm{C}$, followed by a wash with $100 \%$ ethanol, and stored in $100 \%$ ethanol until paraffin embedding and sectioning. Colon sections were incubated with 500 ng Alexa Fluor 488-conjugated EUB338 probe $\left(5^{\prime}\right.$-GCTGCCTCCCGTAGGAGT- $\left.3^{\prime}\right)$ for detection of all bacteria or Cy5-conjugated Muc1437 probe (5'-CCTTGCGGTTGGCTTCAGAT $\left.-3^{\prime}\right)^{43}$ for detection of A. muciniphila in $50 \mu$ l of hybridization buffer (20 mu Tris $\mathrm{HCl}, \mathrm{pH} 7.4,0.9 \mathrm{M} \mathrm{NaCl}, 0.1 \% \mathrm{SDS}, 20 \%$ formamide) at $50{ }^{\circ} \mathrm{C}$ for $3 \mathrm{~h}$. Slides were incubated for $10 \mathrm{~min}$ with FISH washing buffer $(20 \mathrm{~mm}$ Tris- $\mathrm{HCl}$, $\mathrm{pH} 7.4,0.9 \mathrm{M} \mathrm{NaCl}$ ) preheated to $50^{\circ} \mathrm{C}$, and washed three times with PBS, followed by incubation with $10 \mu \mathrm{g} \mathrm{ml}^{-1}$ 4',6-diamidino-2phenylindole in PBS in the dark for $10 \mathrm{~min}$ at room temperature. Then slide were washed with distilled water, allowed to dry, and mounted in SlowFade Diamond Antifade Mountant (Life Technologies).

C. rodentium and $E$. coli 0157: $\mathrm{H} 7$ infection. C. rodentium strain DBS100 (ATCC51459; American Type Culture Collection, Manassas, VA) and E. coli O157:H7 (ATCC700728; American Type Culture Collection) were prepared by shaking bacteria overnight at $37^{\circ} \mathrm{C}$ in Luria-Bertani broth. The concentrations of bacteria were determined by measuring absorbance at optical density 600 . Bacteria were serially diluted and each inoculation culture was plated to confirm the colonyforming units. Mice were fasted for $8 \mathrm{~h}$ before oral inoculation with $1 \times 10^{9}$ colony-forming unit $C$. rodentium or E. coli $\mathrm{O} 157: \mathrm{H} 7$ in a total volume of $200 \mu \mathrm{l}$ per mouse. Feces were collected for bacterial DNA analysis or colony-forming unit counts.

DSS-induced intestinal inflammation. Mice were fed with water containing 2.0-4.0\% DSS (molecular weight 36000-50000; MP Biomedicals, Santa Ana, CA) for 5-7 days. After the DSS treatment, mice were maintained on regular water. Body weight, stool softness, and blood in the rectum or fur were recorded daily. Disease was scored as previously described ${ }^{44}$ : (1) weight loss (no change $=0 ;<5 \%=1 ; 6$ $10 \%=2 ; 11-20 \%=3 ;>20 \%=4$ ); (2) feces (normal $=0$; pasty, semiformed $=2$; liquid, sticky, or unable to defecate after $5 \mathrm{~min}=4$ ); (3) blood (no blood $=0$; visible blood in rectum $=1$; visible blood on fur $=2)$; and (4) general appearance $($ normal $=0$; piloerection $=1$; lethargy and piloerection $=2$; motionless $=4$ ).

Fecal microbiota transplantation. Groups of male donor mice $(n=3)$ were used for microbiota transplantation. Fresh feces $(0.5 \mathrm{~g})$ was collected within $30 \mathrm{~min}$. The feces were then immediately resuspended in $1 \mathrm{ml}$ sterile PBS and centrifuged at $400 \times g$ for $5 \mathrm{~min}$, to remove large particles from bacteria. The supernatants were collected and orally gavaged into 8 - to 10 -week-old recipient mice. Th reecipient mice were pre-treated with broad-spectrum antibiotics in drinking water for 7 days before switching to autoclaved drinking water for 2 days and then were transplanted with fecal microbiota. Five days post microbiota transplantation, the feces from recipient mice were collected and analyzed for microbiota composition.

Detection of Muc2-bound bacteria. Feces were collected in tubes containing cold PBS. After weights measurement, PBS was added to make 10-fold diluted suspensions (final $1 \mathrm{ml}$ ). PBS-diluted feces were mixed well and centrifuged at $400 \times g$ for $5 \mathrm{~min}$, to remove large particles from bacteria. The supernatants $(500-1000 \mu \mathrm{l})$ were transferred to a new tube and incubated with $2 \mu \mathrm{g}$ of anti-Muc2 antibody (Santa Cruz; sc-15334) or control antibody for $1 \mathrm{~h}$ at $4{ }^{\circ} \mathrm{C}$. The supernatants were then incubated with $20 \mu \mathrm{l}$ of resuspended Protein A/G PLUS-Agarose (Santa Cruz) at $4{ }^{\circ} \mathrm{C}$ on a rotating device for $4 \mathrm{~h}$. The immunoprecipitates were collected by centrifugation at $200 \times g$ for $5 \mathrm{~min}$ at $4{ }^{\circ} \mathrm{C}$. The pellets were washed six to eight times with cold PBS. After the final wash, the pellets were analyzed for Muc2bound bacteria using Stool Genomic DNA Kit (CWBIO).

Statistical analyses. Statistical analysis, excluding microbiome, was performed using Prism 6.0 (GraphPad Software, San Diego, CA). Statistical analyses were performed with averages of biological replicates. Comparisons for animal experiments between two groups without a distribution assumption were analyzed using a two-tailed MannWhitney test. Statistical significance was defined as $P$-value of $<0.05$.

SUPPLEMENTARY MATERIAL is linked to the online version of the paper at http://www.nature.com/mi

\section{ACKNOWLEDGMENTS}

We thank R. Kageyama (Koyto University) for Hes ${ }^{f / f l}$ mice. This research was supported by Ministry of Science and Technology of China National Key Research Project 2015CB943201 (X.H.), National Natural Science Foundation of China $(81422019,81661130161$, and 91642115 to X.H., and 81601443 to X.K.G.), funds from Tsinghua-Peking Center for Life Sciences (X.H. and X.K.G.), Institute for Immunology at Tsinghua University (X.H.), Beijing Municipal Natural Science Foundation (7164267 to X.K.G.), and China Postdoctoral Science Foundation (X.K.G.).

\section{AUTHOR CONTRIBUTIONS}

X.K.G. designed research, performed experiments, analyzed data, and wrote the manuscript. J.O. performed some of the antibiotics-treatmentrelated experiments. S.L. prepared mice for some experiments. X.Z. provided advices on sequencing experiments and on bioinformatic analysis. X.H. conceptualized the project, designed research, supervised experiments, and wrote the manuscript.

\section{DISCLOSURE}

The authors declared no conflict of interest.

(c) 2018 Society for Mucosal Immunology

\section{REFERENCES}

1. Peterson, L. W. \& Artis, D. Intestinal epithelial cells: regulators of barrier function and immune homeostasis. Nat. Rev. Immunol. 14, 141-153 (2014). 
2. Gallo, R. L. \& Hooper, L. V. Epithelial antimicrobial defence of the skin and intestine. Nat. Rev. Immunol. 12, 503-516 (2012).

3. Johansson, M. E. \& Hansson, G. C. Immunological aspects of intestinal mucus and mucins. Nat. Rev. Immunol. 16, 639-649 (2016).

4. Johansson, M. E., Phillipson, M., Petersson, J., Velcich, A., Holm, L. \& Hansson, G. C. The inner of the two Muc2 mucin-dependent mucus layers in colon is devoid of bacteria. Proc. Natl Acad. Sci. USA 105, 15064-15069 (2008).

5. Rooks, M. G. \& Garrett, W. S. Gut microbiota, metabolites and host immunity. Nat. Rev. Immunol. 16, 341-352 (2016).

6. Petnicki-Ocwieja, T., Hrncir, T., Liu, Y. J., Biswas, A., Hudcovic, T. \& Tlaskalova-Hogenova, $\mathrm{H}$. et al. Nod2 is required for the regulation of commensal microbiota in the intestine. Proc. Natl Acad. Sci. USA 106, 15813-15818 (2009).

7. Nieuwenhuis, E. E., Matsumoto, T., Lindenbergh, D., Willemsen, R., Kaser, A. \& Simons-Oosterhuis, Y. et al. Cd1d-dependent regulation of bacterial colonization in the intestine of mice. J. Clin. Invest. 119, 1241-1250 (2009).

8. Salzman, N. H., Hung, K., Haribhai, D., Chu, H., Karlsson-Sjoberg, J. \& Amir, E. et al. Enteric defensins are essential regulators of intestinal microbial ecology. Nat. Immunol. 11, 76-83 (2010).

9. Elinav, E., Strowig, T., Kau, A. L., Henao-Mejia, J., Thaiss, C. A. \& Booth, C. J. et al. NLRP6 inflammasome regulates colonic microbial ecology and risk for colitis. Cell 145, 745-757 (2011).

10. Donaldson, G. P., Lee, S. M. \& Mazmanian, S. K. Gut biogeography of the bacterial microbiota. Nat. Rev. Microbiol. 14, 20-32 (2016).

11. Okai, S., Usui, F., Yokota, S., Hori, I. Y., Hasegawa, M. \& Nakamura, T. et al. High-affinity monoclonal IgA regulates gut microbiota and prevents colitis in mice. Nat. Microbiol. 1, 16103 (2016).

12. Ruan, J. W., Statt, S., Huang, C. T., Tsai, Y. T., Kuo, C. C. \& Chan, H. L. et al. Dual-specificity phosphatase 6 deficiency regulates gut microbiome and transcriptome response against diet-induced obesity in mice. Nat. Microbiol. 2, 16220 (2016).

13. Dethlefsen, L. \& Relman, D. A. Incomplete recovery and individualized responses of the human distal gut microbiota to repeated antibiotic perturbation. Proc. Natl Acad. Sci. USA 108 (Suppl 1), 4554-4561 (2011).

14. Kobayashi, T. \& Kageyama, R. Expression dynamics and functions of Hes factors in development and diseases. Curr. Top. Dev. Biol. 110, 263-283 (2014).

15. Jensen, J., Pedersen, E. E., Galante, P., Hald, J., Heller, R. S. \& Ishibashi, M. et al. Control of endodermal endocrine development by Hes-1. Nat. Genet. 24, 36-44 (2000).

16. Suzuki, K., Fukui, H., Kayahara, T., Sawada, M., Seno, H. \& Hiai, H. et al. Hes1-deficient mice show precocious differentiation of Paneth cells in the small intestine. Biochem. Biophys. Res. Commun. 328, 348-352 (2005).

17. van der Flier, L. G. \& Clevers, H. Stem cells, self-renewal, and differentiation in the intestinal epithelium. Annu. Rev. Physiol. 71, 241-260 (2009).

18. Noah, T. K. \& Shroyer, N. F. Notch in the intestine: regulation of homeostasis and pathogenesis. Annu. Rev. Physiol. 75, 263-288 (2013).

19. Ueo, T., Imayoshi, I., Kobayashi, T., Ohtsuka, T., Seno, H. \& Nakase, H. et al. The role of Hes genes in intestinal development, homeostasis and tumor formation. Development 139, 1071-1082 (2012).

20. Hu, X., Chung, A. Y., Wu, I., Foldi, J., Chen, J. \& Ji, J. D. et al. Integrated regulation of Toll-like receptor responses by Notch and interferon-gamma pathways. Immunity 29, 691-703 (2008).

21. Su, X., Yu, Y., Zhong, Y., Giannopoulou, E. G., Hu, X. \& Liu, H. et al. Interferon-gamma regulates cellular metabolism and mRNA translation to potentiate macrophage activation. Nat. Immunol. 16, 838-849 (2015).

22. Shang, Y., Coppo, M., He, T., Ning, F., Yu, L. \& Kang, L. et al. The transcriptional repressor Hes1 attenuates inflammation by regulating transcription elongation. Nat. Immunol. 17, 930-937 (2016).
23. Shang, Y., Smith, S. \& Hu, X. Role of Notch signaling in regulating innate immunity and inflammation in health and disease. Protein Cell 7, 159-174 (2016).

24. Xu, H. et al. Notch-RBP-J signaling regulates the transcription factor IRF8 to promote inflammatory macrophage polarization. Nat. Immunol. 13, 642-650 (2012).

25. Buffie, C. G. \& Pamer, E. G. Microbiota-mediated colonization resistance against intestinal pathogens. Nat. Rev. Immunol. 13, 790-801 (2013).

26. Fagarasan, S., Kawamoto, S., Kanagawa, O. \& Suzuki, K. Adaptive immune regulation in the gut: $T$ cell-dependent and $T$ cell-independent IgA synthesis. Annu. Rev. Immunol. 28, 243-273 (2010).

27. De Obaldia, M. E. \& Bhandoola, A. Transcriptional regulation of innate and adaptive lymphocyte lineages. Annu. Rev. Immunol. 33, 607-642 (2015).

28. Sonnenburg, J. L. et al. Glycan foraging in vivo by an intestine-adapted bacterial symbiont. Science 307, 1955-1959 (2005).

29. Berry, D. et al. Host-compound foraging by intestinal microbiota revealed by single-cell stable isotope probing. Proc. Natl Acad. Sci. USA 110, 4720-4725 (2013).

30. Larsson, J. M., Karlsson, H., Sjovall, H. \& Hansson, G. C. A complex, but uniform O-glycosylation of the human MUC2 mucin from colonic biopsies analyzed by nanoLC/MSn. Glycobiology 19, 756-766 (2009).

31. Png, C. W. et al. Mucolytic bacteria with increased prevalence in IBD mucosa augment in vitro utilization of mucin by other bacteria. Am. J. Gastroenterol. 105, 2420-2428 (2010).

32. Lee, S. M., Donaldson, G. P., Mikulski, Z., Boyajian, S., Ley, K. \& Mazmanian, S. K. Bacterial colonization factors control specificity and stability of the gut microbiota. Nature 501, 426-429 (2013).

33. Rakoff-Nahoum, S., Paglino, J., Eslami-Varzaneh, F., Edberg, S. \& Medzhitov, R. Recognition of commensal microflora by toll-like receptors is required for intestinal homeostasis. Cell 118, 229-241 (2004).

34. Pope, J. L. et al. Claudin-1 regulates intestinal epithelial homeostasis through the modulation of Notch-signalling. Gut 63, 622-634 (2014).

35. Guilmeau, S. et al. Intestinal deletion of Pofut1 in the mouse inactivates notch signaling and causes enterocolitis. Gastroenterology 135, 849-860. 860 e841-846 (2008).

36. Imayoshi, I., Shimogori, T., Ohtsuka, T. \& Kageyama, R. Hes genes and neurogenin regulate non-neural versus neural fate specification in the dorsal telencephalic midline. Development 135, 2531-2541 (2008).

37. Vaishnava, S. et al. The antibacterial lectin Reglllgamma promotes the spatial segregation of microbiota and host in the intestine. Science 334 , 255-258 (2011).

38. Magoc, T. \& Salzberg, S. L. FLASH: fast length adjustment of short reads to improve genome assemblies. Bioinformatics 27, 2957-2963 (2011).

39. Caporaso, J. G. et al. QIIME allows analysis of high-throughput community sequencing data. Nat. Methods 7, 335-336 (2010).

40. Edgar, R. C., Haas, B. J., Clemente, J. C., Quince, C. \& Knight, R. UCHIME improves sensitivity and speed of chimera detection. Bioinformatics 27 , 2194-2200 (2011).

41. Wang, Q., Garrity, G. M., Tiedje, J. M. \& Cole, J. R. Naive Bayesian classifier for rapid assignment of rRNA sequences into the new bacterial taxonomy. Appl. Environ. Microbiol. 73, 5261-5267 (2007).

42. Johansson, M. E. \& Hansson, G. C. Preservation of mucus in histological sections, immunostaining of mucins in fixed tissue, and localization of bacteria with FISH. Methods Mol. Biol. 842, 229-235 (2012).

43. Derrien, M., Collado, M. C., Ben-Amor, K., Salminen, S. \& de Vos, W. M. The Mucin degrader Akkermansia muciniphila is an abundant resident of the human intestinal tract. Appl. Environ. Microbiol. 74, 1646-1648 (2008).

44. Alenghat, T. et al. Histone deacetylase 3 coordinates commensal-bacteriadependent intestinal homeostasis. Nature 504, 153-157 (2013). 